\title{
Green's Functions for Mixed Boundary Value Problems in Regions of Irregular Shape
}

\author{
Y.A. Melnikov and M.Y. Melnikov \\ Department of Mathematical Sciences \\ Middle Tennessee State University \\ Murfreesboro, TN 37132, U.S.A.
}

\begin{abstract}
A semi-analytic approach is applied to the construction of Green's functions and matrices of Green's type for Laplace and Klein-Gordon equation in two dimensions. Mixed boundary value problems posed in multiply connected regions are considered. Statements of the problems are complicated by intricate geometry of the regions and different types of boundary conditions imposed on different fragments of the boundary. The approach is based on a combination of the Green's function method and the method of functional equations. Kernels of resolving potentials representing the regular components of the Green's functions to be constructed are built with Green's functions obtained for regions of a standard shape.

Keywords: Green's function; Laplace equation; Klein-Gordon equation; Mixed boundary value problems; Regions of irregular shape.
\end{abstract}

\section{Introduction}

There exists an opinion within the engineering community that Green's functions for applied partial differential equations do not represent a competitive and convenient instrument in the realm of practical computer implementations. The present project is a part of a wider research undertaken by the authors in recent years and having the intention to disprove that opinion. The opponents of the Green's function approach do not dispute its power in the qualitative theory of differential equations. What they are contesting is the computational potential of this approach. One of the arguments that they traditionally adduce is the myth that Green's functions can only be constructed for simply connected regions of a stan- 
dard configuration where the region's boundary is presented by coordinate surfaces (lines) in a single conventional coordinate system. Until recently, this argument was dominant among engineers, but has been critically revised in a series of recent studies [3-9], the results of which call the myth in question.

A semi-analytic technique has earlier been developed [3] that can successfully be utilized for computing Green's functions for a certain class of multiply connected regions of irregular shape. The regions might be filled in with materials whose property functions are piecewise homogeneous. Note that, within this technique, the outer contour of a region is of a standard shape and assumed piecewise smooth, while the inner contours represent smooth curves that do not cross the lines of discontinuity (if any) of the material properties.

Note also that although boundary conditions imposed on the outer contours could be of a mixed type, the technique has only been applicable so far to problems with Dirichlet boundary conditions imposed on the inner contours. The technique assumes that Green's functions or matrices of Green's type for corresponding simply connected regions of standard shape are either available or can routinely be obtained. These Green's functions are used to compile the kernels of some potential representations that are employed to obtain Green's functions for multiply connected regions.

To further extend the technique and to make it readily applicable to real settings in engineering, the present study aims at boundary value problems for multiply connected regions: (i) with other than Dirichlet boundary conditions imposed on inner contours, (ii) for a wider variety of shapes of inner contours, and (iii) with possible intersections of inner contours by the lines of discontinuity of material properties.

A break-through became possible in the construction of Green's functions to mixed boundary value problems for regions of non-standard shape after a score of computer-friendly forms of Green's functions were obtained (see [3]) for simply connected regions of standard configuration. The point is that most of the representations of Green's functions available in literature are inconvenient for computer implementations. As a typical example of a non-computer-friendly representation, we recall the classical double series form

$$
G(x, y ; \xi, \eta)=\frac{4}{a b} \sum_{m, n=1}^{\infty} \frac{\sin (m \pi x / a) \sin (n \pi y / b) \sin (m \pi \xi / a) \sin (n \pi \eta / b)}{(m \pi / a)^{2}+(n \pi / b)^{2}}
$$

of the Green's function of Laplace equation for Dirichlet problem in the rectangle $\{0<x<a, 0<y<b\}$. The series in this representation does not (and cannot) uniformly converge making the above form inconvenient in computations unless some analytical adjustments accelerating the convergence are performed in advance. 


\section{Simply connected regions of standard shape}

We will enlist, in this section, a few mixed (with more than one kind of boundary conditions involved) boundary value problems for the two-dimensional Laplace and Klein-Gordon equation in regions of standard shape that allow readily computable forms of Green's functions. Those will be used in Sections 3 and 4 where mixed problems for regions of irregular configuration are considered. The first of those is

$$
\begin{gathered}
G(x, y ; \xi, \eta)=\frac{1}{2 \pi} \ln \frac{\left|1-e^{p(z-\bar{\zeta})}\right|\left|1-e^{p(z+\zeta)}\right|}{\left|1-e^{p(z-\zeta)}\right|\left|1-e^{p(z+\bar{\zeta})}\right|} \\
+\frac{1}{2 \pi} \ln \frac{\left|1+e^{p(z+\bar{\zeta})}\right|\left|1+e^{p(z-\zeta)}\right|}{\left|1+e^{p(z-\bar{\zeta})}\right|\left|1+e^{p(z+\zeta)}\right|} \\
-\frac{2 \beta}{b} \sum_{n=1,3,5, \ldots} \frac{e^{-\nu(x+\xi)}}{\nu(\nu+\beta)} \sin \nu y \sin \nu \eta, \quad \nu=n p, \quad p=\frac{\pi}{2 b}
\end{gathered}
$$

the Green's function of the mixed boundary value problem

$$
\left.\left(\frac{\partial u}{\partial x}-\beta u\right)\right|_{x=0}=\left.u\right|_{y=0}=\left.\frac{\partial u}{\partial y}\right|_{y=b}=0, \quad|u|_{x=\infty}<\infty, \quad \beta \geq 0
$$

in the semi-strip $\{\operatorname{Re}(z)>0,0<\operatorname{Im}(z)<b\}$. Here $z=(x, y)$ and $\zeta=(\xi, \eta)$ are the observation point and the source point, respectively.

Notice a specific peculiarity of the above statement. It represents a mixed boundary value problem with three different kinds of boundary conditions imposed on three different fragments of the region's boundary.

Two important features make the representation in Eq.(1) effective in computer implementations. First, the singular component of the Green's function (in a form of the fundamental solution of the Laplace equation) is split off explicitly. Indeed, it is contained in the logarithmic term

$$
\frac{1}{2 \pi} \ln \frac{1}{\left|1-e^{p(z-\zeta)}\right|}
$$

and becomes explicit after the exponent $e^{p(z-\zeta)}$ is expanded in Taylor series

$$
e^{p(z-\zeta)}=\sum_{n=0}^{\infty} \frac{p^{n}(z-\zeta)^{n}}{n !}
$$

making the difference $(z-\zeta)$ a common factor in the denominator of (2).

The second important feature making the representation in Eq.(1) computationally effective is that its series uniformly converges at the rate of $1 / n^{2}$. Hence, 
values of the Green's function defined by Eq.(1) can be accurately computed by a direct truncation of the series. Sections 3 and 4 deliver persuasive confirmation of this assertion while we compute Green's functions for a semi-strip with apertures and cut-offs of different configuration and location.

To introduce a matrix of Green's type for a region of standard shape filled in with a piecewise homogeneous material, we consider now a half-plane $\Omega=\{0<$ $r<\infty, 0<\varphi<\pi\}$ composed of two fragments. That is, $\Omega=\Omega_{1} \cup \Omega_{2}$, where $\Omega_{1}=\{0<r<a, 0<\varphi<\pi\}$ and $\Omega_{2}=\{a<r<\infty, 0<\varphi<\pi\}$, and pose the following boundary-contact value problem for functions $u_{1}(r, \varphi)$ and $u_{2}(r, \varphi)$ defined on the regions $\Omega_{1}$ and $\Omega_{2}$, respectively

$$
\begin{gathered}
\frac{1}{r} \frac{\partial}{\partial r}\left(r \frac{\partial u_{1}(r, \varphi)}{\partial r}\right)+\frac{1}{r^{2}} \frac{\partial^{2} u_{1}(r, \varphi)}{\partial \varphi^{2}}=0, \quad(0, a) \times(0, \pi) \\
\frac{1}{r} \frac{\partial}{\partial r}\left(r \frac{\partial u_{2}(r, \varphi)}{\partial r}\right)+\frac{1}{r^{2}} \frac{\partial^{2} u_{2}(r, \varphi)}{\partial \varphi^{2}}=0, \quad(a, \infty) \times(0, \pi) \\
\left|u_{1}(0, \varphi)\right|<\infty, \quad\left|u_{2}(\infty, \varphi)\right|<\infty \\
u_{1}(a, \varphi)=u_{2}(a, \varphi), \quad \frac{\partial u_{1}(a, \varphi)}{\partial r}=\lambda \frac{\partial u_{2}(a, \varphi)}{\partial r} \\
u_{1}(r, 0)=u_{2}(r, 0)=u_{1}(r, \pi)=u_{2}(r, \pi)=0,
\end{gathered}
$$

where $\lambda=\lambda_{2} / \lambda_{1}$ stands for the relative material conductivity, with $\lambda_{1}$ and $\lambda_{2}$ representing the physical properties of the materials with which the regions $\Omega_{1}$ and $\Omega_{2}$, respectively, are filled in.

The matrix of Green's type

$$
G=\left(G_{i j}(r, \varphi ; \rho, \psi)\right)_{i, j=1,2}
$$

of the above problem has been constructed with its entries expressed in terms of elementary functions as

$$
\begin{array}{r}
G_{11}(r, \varphi ; \rho, \psi)=\frac{1}{4 \pi}\left[\ln \frac{r^{2}-2 r \rho \cos (\varphi+\psi)+\rho^{2}}{r^{2}-2 r \rho \cos (\varphi-\psi)+\rho^{2}}\right. \\
\left.-\frac{\lambda-1}{\lambda+1} \ln \frac{a^{4}-2 a^{2} r \rho \cos (\varphi+\psi)+r^{2} \rho^{2}}{a^{4}-2 a^{2} r \rho \cos (\varphi-\psi)+r^{2} \rho^{2}}\right] \\
G_{12}(r, \varphi ; \rho, \psi)=\frac{\lambda}{2 \pi(\lambda+1)} \ln \frac{r^{2}-2 r \rho \cos (\varphi+\psi)+\rho^{2}}{r^{2}-2 r \rho \cos (\varphi-\psi)+\rho^{2}} \\
G_{21}(r, \varphi ; \rho, \psi)=\frac{1}{2 \pi(\lambda+1)} \ln \frac{r^{2}-2 r \rho \cos (\varphi+\psi)+\rho^{2}}{r^{2}-2 r \rho \cos (\varphi-\psi)+\rho^{2}}
\end{array}
$$


and

$$
\begin{gathered}
G_{22}(r, \varphi ; \rho, \psi)=\frac{1}{4 \pi}\left[\ln \frac{r^{2}-2 r \rho \cos (\varphi+\psi)+\rho^{2}}{r^{2}-2 r \rho \cos (\varphi-\psi)+\rho^{2}}\right. \\
\left.+\frac{\lambda-1}{\lambda+1} \ln \frac{a^{4}-2 a^{2} r \rho \cos (\varphi+\psi)+r^{2} \rho^{2}}{a^{4}-2 a^{2} r \rho \cos (\varphi-\psi)+r^{2} \rho^{2}}\right]
\end{gathered}
$$

It is evident that the above matrix is suitable for direct computer implementations. The reader can find a confirmation of this assertion in Sections 3 and 4 where we compute matrices of Green's type for a composed half-plane weakened with apertures and cut-offs.

Consider another boundary-contact value problem

$$
\begin{gathered}
\frac{\partial^{2} u_{i}}{\partial x^{2}}+\frac{\partial^{2} u_{i}}{\partial y^{2}}-k_{i}^{2} u_{i}=0, \quad(x, y) \in \Omega_{i}, \quad(i=1,2) \\
\frac{\partial u_{1}(-a, y)}{\partial x}-\beta u_{1}(-a, y)=0, \quad\left|u_{2}(\infty, y)\right|<\infty \\
u_{1}(0, y)=u_{2}(0, y), \quad \lambda_{1} \frac{\partial u_{1}(0, y)}{\partial x}=\lambda_{2} \frac{\partial u_{2}(0, y)}{\partial x} \\
u_{i}(x, 0)=0, \quad \frac{\partial u_{i}(x, b)}{\partial y}=0, \quad(i=1,2)
\end{gathered}
$$

for Klein-Gordon equations that governs two functions: $u_{1}=u_{1}(x, y)$ defined on the fragment $\Omega_{1}=\{-a<x<0,0<y<b\}$ and $u_{2}=u_{2}(x, y)$ defined on $\Omega_{2}=\{0<$ $x<\infty, 0<y<b\}$ compiling the semi-infinite strip $\Omega=\{-a<x<\infty, 0<y<b\}$. Here the parameters $\lambda_{1}$ and $\lambda_{2}$ specify the conductive properties of the materials of which the fragments of $\Omega$ are filled in.

Entries $G_{i j}(x, y ; \xi, \eta),(i, j=1,2)$ of the matrix of Green's type for the above setting are obtained as

$$
G_{i j}(x, y ; \xi, \eta)=\frac{2}{b} \sum_{n=1}^{\infty} \frac{1}{\Delta} g_{i j}^{n}(x, \xi) \sin \nu y \sin \nu \eta, \quad \nu=\frac{(2 n-1) \pi}{2 b}
$$

where

$$
\Delta=\left(h_{1}+\lambda h_{2}\right)\left(h_{1}+\beta\right) e^{2 h_{1} a}+\left(h_{1}-\lambda h_{2}\right)\left(h_{1}-\beta\right),
$$

the relative material conductivity is defined with $\lambda=\lambda_{2} / \lambda_{1}, h_{i}=\sqrt{\nu^{2}+k_{i}^{2}}, \quad(i=$ $1,2)$, while the coefficients $g_{i j}^{n}(x, \xi)$ are defined as

$$
\begin{aligned}
g_{11}^{n}(x, \xi) & =\frac{1}{2 h_{1}}\left\{\left(h_{1}+\beta\right)\left[\left(h_{1}+\lambda h_{2}\right) e^{-h_{1}|x-\xi|}+\left(h_{1}-\lambda h_{2}\right) e^{-h_{1}(x+\xi)}\right] e^{2 h_{1} a}\right. \\
& \left.+\left(h_{1}-\beta\right)\left[\left(h_{1}+\lambda h_{2}\right) e^{-h_{1}(x+\xi)}+\left(h_{1}-\lambda h_{2}\right) e^{-h_{1}|x-\xi|}\right]\right\}
\end{aligned}
$$




$$
\begin{aligned}
& g_{12}^{n}(x, \xi)=\lambda\left[\left(h_{1}+\beta\right) e^{h_{1}(2 a+x)}+\left(h_{1}-\beta\right) e^{-h_{1} x}\right] e^{-h_{2} \xi} \\
& g_{21}^{n}(x, \xi)=\left[\left(h_{1}+\beta\right) e^{h_{1}(2 a+\xi)}+\left(h_{1}-\beta\right) e^{-h_{1} \xi}\right] e^{-h_{2} x}
\end{aligned}
$$

and

$$
\begin{aligned}
g_{22}^{n}(x, \xi) & =\frac{1}{2 h_{2}}\left\{\left(h_{1}+\beta\right)\left[\left(h_{1}+\lambda h_{2}\right) e^{-h_{2}|x-\xi|}-\left(h_{1}-\lambda h_{2}\right) e^{-h_{2}(x+\xi)}\right] e^{2 h_{1} a}\right. \\
& \left.+\left(h_{1}-\beta\right)\left[\left(h_{1}+\lambda h_{2}\right) e^{-h_{2}(x+\xi)}-\left(h_{1}-\lambda h_{2}\right) e^{-h_{2}|x-\xi|}\right]\right\}
\end{aligned}
$$

Another matrix of Green's type

$$
G=\left(G_{i j}(x, y ; \xi, \eta)\right)_{i, j=1,3}
$$

has been constructed for the following boundary-contact value problem

$$
\begin{gathered}
\frac{\partial^{2} u_{i}}{\partial x^{2}}+\frac{\partial^{2} u_{i}}{\partial y^{2}}=0, \quad(x, y) \in \Omega_{i}, \quad(i=1,2,3) \\
\left|u_{1}(-\infty, y)\right|<\infty, \quad\left|u_{3}(\infty, y)\right|<\infty \\
u_{1}(-a, y)=u_{2}(-a, y), \quad \lambda_{1} \frac{\partial u_{1}(-a, y)}{\partial x}=\lambda_{2} \frac{\partial u_{2}(-a, y)}{\partial x} \\
u_{2}(a, y)=u_{2}(a, y), \quad \lambda_{2} \frac{\partial u_{2}(a, y)}{\partial x}=\lambda_{3} \frac{\partial u_{3}(a, y)}{\partial x} \\
u_{i}(x, 0)=0, \quad \frac{\partial u_{i}(x, b)}{\partial y}=0, \quad(i=1,2,3)
\end{gathered}
$$

posed for the functions $u_{i}=u_{i}(x, y),(i=1,2,3)$ each of which is defined on the corresponding fragment $\Omega_{1}=\{-\infty<x<-a, 0<y<b\}, \Omega_{2}=\{-a<x<a, 0<y<$ $b\}$, and $\Omega_{3}=\{a<x<\infty, 0<y<b\}$ of the infinite strip $\Omega=\{-\infty<x<\infty, 0<y<b\}$. Conductive properties of the materials, of which the fragments $\Omega_{i}$ are filled with, are specified by the constants $\lambda_{i},(i=1,2,3)$.

Entries of the matrix of Green's type $G$ are found in this case as

$$
G_{i j}(x, y ; \xi, \eta)=\frac{1}{b} \sum_{n=1}^{\infty} \frac{1}{\nu \Delta^{*}} g_{i j}^{n}(x, \xi) \sin \nu y \sin \nu \eta, \quad \nu=\frac{(2 n-1) \pi}{2 b}
$$

where

$$
\Delta^{*}=\left(1-\Lambda_{1}\right)\left(1-\Lambda_{2}\right)+\left(1+\Lambda_{1}\right)\left(1+\Lambda_{2}\right) e^{4 \nu a},
$$

with the relative material conductivities defined with $\Lambda_{1}=\lambda_{2} / \lambda_{1}$ and $\Lambda_{2}=\lambda_{3} / \lambda_{2}$, while the coefficients $g_{i j}^{n}(x, \xi)$ read as

$$
g_{11}^{n}(x, \xi)=\left[\left(1+\Lambda_{1}\right)\left(1-\Lambda_{2}\right)+\left(1-\Lambda_{1}\right)\left(1+\Lambda_{2}\right) e^{4 \nu a}\right] e^{\nu(x+\xi+2 a)}+\Delta^{*} e^{-\nu|x-\xi|}
$$




$$
\begin{gathered}
g_{12}^{n}(x, \xi)=2 \Lambda_{1}\left[\left(1-\Lambda_{2}\right) e^{\nu(\xi-a)}+\left(1+\Lambda_{2}\right) e^{\nu(a-\xi)}\right] e^{\nu(3 a+x)} \\
g_{13}^{n}(x, \xi)=4 \Lambda_{1} \Lambda_{2} e^{\nu(4 a+x-\xi)} \\
g_{21}^{n}(x, \xi)=2\left[\left(1-\Lambda_{2}\right) e^{\nu(x+a)}+\left(1+\Lambda_{2}\right) e^{\nu(3 a-x)}\right] e^{\nu(a+\xi)} \\
g_{22}^{n}(x, \xi)=\left(\Lambda_{1}+1\right)\left[\left(1-\Lambda_{2}\right) e^{\nu(x+\xi+2 a)}+\left(1+\Lambda_{2}\right) e^{\nu(4 a-|x-\xi|)}\right] \\
+\left(\Lambda_{1}-1\right)\left[\left(1-\Lambda_{2}\right) e^{\nu(x-\xi)}+\left(1+\Lambda_{2}\right) e^{\nu(2 a-x-\xi)}\right] \\
g_{23}^{n}(x, \xi)=2 \Lambda_{2}\left[\left(1+\Lambda_{1}\right) e^{\nu(3 a+x)}+\left(\Lambda_{1}-1\right) e^{\nu(a-x)}\right] e^{\nu(a-\xi)} \\
g_{31}^{n}(x, \xi)=4 e^{\nu(4 a-x+\xi)} \\
g_{32}^{n}(x, \xi)=2\left[\left(1+\Lambda_{1}\right) e^{\nu(3 a+\xi)}+\left(\Lambda_{1}-1\right) e^{\nu(a-\xi)}\right] e^{\nu(a-x)}
\end{gathered}
$$

and

$$
g_{33}^{n}(x, \xi)=\left[\left(\Lambda_{1}-1\right)\left(\Lambda_{2}+1\right)+\left(\Lambda_{1}+1\right)\left(\Lambda_{2}-1\right) e^{4 \nu a}\right] e^{\nu(2 a-x-\xi)}+\Delta^{*} e^{-\nu|x-\xi|}
$$

Note that the series representing the diagonal etries $G_{i i}$ of $G$ converge at a low rate of $1 / n$ and are, therefore, ineffictive in computer implementations. To enhance the computability of the entry $G_{11}$, for example, after some transformations we rewrite its coefficient in the form

$$
\begin{gathered}
\frac{g_{11}^{n}(x, \xi)}{(2 n-1) \Delta^{*}}=\frac{1}{2 n-1} e^{-\nu|x-\xi|} \\
+\frac{\left(1-\Lambda_{1}\right)}{(2 n-1)\left(1+\Lambda_{1}\right)} e^{\nu(x+\xi+2 a)}+\frac{4 \Lambda_{1}\left(1-\Lambda_{2}\right) e^{\nu(x+\xi+2 a)}}{(2 n-1)\left(1+\Lambda_{1}\right) \Delta^{*}}
\end{gathered}
$$

This yields

$$
\begin{gathered}
G_{11}(x, y ; \xi, \eta)=\frac{1}{\pi} \sum_{n=1}^{\infty} \frac{e^{-\nu|x-\xi|}}{2 n-1}[\cos \nu(y-\eta)-\cos \nu(y+\eta)] \\
+\frac{\left(1-\Lambda_{1}\right)}{\pi\left(1+\Lambda_{1}\right)} \sum_{n=1}^{\infty} \frac{e^{\nu(x+\xi+2 a)}}{2 n-1}[\cos \nu(y-\eta)-\cos \nu(y+\eta)] \\
+\frac{4 \Lambda_{1}\left(1-\Lambda_{2}\right)}{\pi\left(1+\Lambda_{1}\right)} \sum_{n=1}^{\infty} \frac{e^{\nu(x+\xi+2 a)}}{(2 n-1) \Delta^{*}}[\cos \nu(y-\eta)-\cos \nu(y+\eta)]
\end{gathered}
$$

Remind that both $(x, y)$ and $(\xi, \eta)$ in $G_{11}(x, y ; \xi, \eta)$ belong to $\Omega_{1}$. This implies that both variables $x$ and $\xi$ never top the value of $-a$ making the last of the above 
series convergent at an extremely high rate and convenient, therefore, in practical implementations in its carrent form. As to the first two series, they are completely summable. Upon performing some algebra, the entry $G_{11}(x, y ; \xi, \eta)$ is ultimately rewritten in a computer-friendly form as

$$
\begin{gathered}
G_{11}(x, y ; \xi, \eta)=\frac{1}{2 \pi}\left\{\ln \frac{\left|1+e^{p(z-\zeta)}\right|\left|1-e^{p(z-\bar{\zeta})}\right|}{\left|1-e^{p(z-\zeta)}\right|\left|1+e^{p(z-\bar{\zeta})}\right|}\right. \\
+\frac{\left(1-\Lambda_{1}\right)}{\left(1+\Lambda_{1}\right)} \ln \frac{\left|1+e^{p\left(z_{1}+\overline{\zeta_{1}}\right)}\right|\left|1-e^{p\left(z_{1}+\zeta_{1}\right)}\right|}{\left|1-e^{p\left(z_{1}+\overline{\zeta_{1}}\right)}\right|\left|1+e^{p\left(z_{1}+\zeta_{1}\right)}\right|} \\
\left.+\frac{16 \Lambda_{1}\left(1-\Lambda_{2}\right)}{\left(1+\Lambda_{1}\right)} \sum_{n=1}^{\infty} \frac{e^{\nu(x+\xi+2 a)}}{(2 n-1) \Delta^{*}} \sin \nu y \sin \nu \eta\right\}, \quad p=\frac{\pi}{2 b}
\end{gathered}
$$

where the following compex variables

$$
z=x+i y, \quad \zeta=\xi+i \eta, \quad z_{1}=(x+a)+i y, \quad \zeta_{1}=(\xi+a)+i \eta
$$

are introduced for compactness, and the bar on a variable means the conjugate.

The other diagonal entries $G_{22}(x, y ; \xi, \eta)$ and $G_{33}(x, y ; \xi, \eta)$ also reduce to a computer-friendly form by using similar transformations.

Later in Sections 3 and 4, the functions and matrices of Green's type enlisted in this Section will be employed to handle mixed boundary value problems for simply and multiply connected regions of irregular configuration.

\section{Simply connected regions of irregular shape}

To advocate an approach that we propose for the computing of Green's functions for mixed boundary value problems in simply connected regions of irregular configuration and to show its potential, we consider, in this section, a number of illustrative examples.

Example 3.1: Consider the region $\Omega=\{0<x<b, x<y<b\}$ representing an isosceles right triangle. Let the following boundary conditions

$$
\left.\left(\frac{\partial u}{\partial x}-\beta u\right)\right|_{x=0}=\left.u\right|_{y=x}=\left.\frac{\partial u}{\partial y}\right|_{y=b}=0, \quad \beta \geq 0
$$

be imposed on its contour.

Fixing the source point $\left(\xi^{*}, \eta^{*}\right)$ inside $\Omega$, we express the Green's function $\Gamma\left(x, y ; \xi^{*}, \eta^{*}\right)$ of the boundary value problem that appeared in Eq.(9) for the Laplace equation in the form

$$
\Gamma\left(x, y ; \xi^{*}, \eta^{*}\right)=G\left(x, y ; \xi^{*}, \eta^{*}\right)+G^{*}(x, y),
$$


where $G\left(x, y ; \xi^{*}, \eta^{*}\right)$ represents the Green's function for the semi-infinite strip, presented earlier by Eq.(1) in Section 2.

It is evident that, due to the presence of the component $G\left(x, y ; \xi^{*}, \eta^{*}\right)$ in Eq. (10), $\Gamma\left(x, y ; \xi^{*}, \eta^{*}\right)$ possesses the logarithmic singularity at $(x, y)=\left(\xi^{*}, \eta^{*}\right)$. On the other hand, for $\Gamma\left(x, y ; \xi^{*}, \eta^{*}\right)$ to be harmonic at any point $(x, y) \neq\left(\xi^{*}, \eta^{*}\right)$ in $\Omega$ and to satisfy the boundary conditions imposed on the fragments $x=0$ and $y=b$, the additive component $G^{*}(x, y)$ in Eq.(10) ought to be harmonic everywhere in $\Omega$ and also satisfy these boundary conditions. This means that for $\Gamma\left(x, y ; \xi^{*}, \eta^{*}\right)$ to satisfy the boundary condition imposed by Eq.(9) on the segment $y=x$, the component $G^{*}(x, y)$ ought to compensate the values of $G\left(x, x ; \xi^{*}, \eta^{*}\right)$ on $y=x$. That is

$$
G^{*}(x, x)=-G\left(x, x ; \xi^{*}, \eta^{*}\right)
$$

In compliance with our algorithm based on the Green's function modification [3] of the method of functional equations [2], we look for $G^{*}(x, y)$ in a form of the modified potential representation

$$
G^{*}(x, y)=\int_{F} G(x, y ; \xi, \eta) \mu(\xi, \eta) d F(\xi, \eta),
$$

where $F$ is a segment of the straight line $y=x-\varepsilon$ (a fictitious contour) ranging from 0 to $b$. Clearly, this segment is parallel to the fragment $y=x$ of the contour of $\Omega$ and is located outside of $\Omega$. Notice that $\varepsilon$ represents a regularizing parameter in our algorithm.

Since the Green's function $G(x, y ; \xi, \eta)$ is the kernel of the integral representation in Eq.(12), the component $G^{*}(x, y)$ is harmonic in $\Omega$ and satisfies the boundary conditions imposed on the fragments $x=0$ and $y=b$. Whereas, when the condition in (11) is satisfied, we come up with the following functional equation

$$
-G\left(x, x ; \xi^{*}, \eta^{*}\right)=\int_{F} G(x, x ; \xi, \eta) \mu(\xi, \eta) d F(\xi, \eta)
$$

in the density function $\mu(\xi, \eta)$.

Since the sets of observation and source points never overlap in (13), its numerical solution is not a problem. The only important issue is a choice of the parameter $\varepsilon$ that provides the regularizing effect in the numerical algorithm. This issue outgh to be handled on a case-by-case basis. After the density function $\mu(\xi, \eta)$ is found, the representation in (10) is used to compute the profile $\Gamma\left(x, y ; \xi^{*}, \eta^{*}\right)$ of the Green's function in $\Omega$.

In Fig. 1 we depict a contourplot of $\Gamma\left(x, y ; \xi^{*}, \eta^{*}\right)$ where the parameters in the statement were chosen as $b=1$ and $\beta=5.0$, while the source point $\left(\xi^{*}, \eta^{*}\right)$ was 
fixed at $(0.3,0.7)$. The series in (1) has been truncated at $N=100$. The estimate of its remainder obtained in [4] suggests that such a truncation allows to attain the accuracy level of $99.9 \%$ in computing values of $G(x, y ; \xi, \eta)$. The regularizing value of the parameter $\varepsilon$ was, in this case, found as 0.05 . The functional equation in (13) was numerically solved by using the standard trapezoidal rule with uniform partition $(m=20)$ of the fictitious contour $F$.

Example 3.2: As another illustrative example, consider a region $\Omega$ bounded with the straight line segments: $x=0, y=0, y=1$ and the quarter-circle $C=$ $\left\{(x-1)^{2}+(y-1)^{2}=1, x>1, y<1\right\}$. Fig. 2 brings the profile $\Gamma(x, y ; 1.0,0.5)$ of the Green's function of the Laplace equation for the problem

$$
\frac{\partial u}{\partial x}-\left.\beta u\right|_{x=0}=\left.u\right|_{C}=\left.u\right|_{y=0}=\left.\frac{\partial u}{\partial y}\right|_{y=1}=0,
$$

posed in $\Omega$. Same algorithm, as described in the previous example, was employed. The Green's function $G(x, y ; \xi, \eta)$ as of Eq.(1), with $b=1$ and $\beta=1$, was used for the first additive component in Eq.(10) and to form the kernel of the resolving potential in Eq.(12). The functional equation

$$
-G\left(x, 1-\sqrt{1-(x-1)^{2}} ; \xi^{*}, \eta^{*}\right)=\int_{F} G\left(x, 1-\sqrt{1-(x-1)^{2}} ; \xi, \eta\right) \mu(\xi, \eta) d F(\xi, \eta)
$$

arises when the boundary condition imposed on the $C$ part of the contour is satisfied. Regularizing parameters were found as: $N=100, m=20, \varepsilon=0.03$, and the fictitious contour is $F=\left\{(x-1)^{2}+(y-1)^{2}=(1+\varepsilon)^{2}, x>1, y<1\right\}$.

Example 3.3: To show that our algorithm can be adjusted to boundarycontact value problems posed on simply connected regions of irregular configuration, we consider a region $\Omega$ bounded with the straight line segments $x=-2$ and $y=0$, the quarter-circle $C=\left\{x^{2}+(y-\pi)^{2}=\pi^{2}\right\}$ (with $0<x, y<\pi$ ), and the line segment $y=\pi$. By the interface $x=0$, the region $\Omega$ brakes onto two subregions: the rectangle $\Omega_{1}=\{-2<x<0,0<y<\pi\}$ and the quarter-disc $\Omega_{2}$, with radius $\pi$, centered at $(0, \pi)$. Each of the subregions is filled in with a material of different conductive property specified with $\lambda_{1}$ and $\lambda_{2}$, respectively.

The following boundary-contact value problem for Klein-Gordon equations

$$
\begin{gathered}
\frac{\partial^{2} u_{i}}{\partial x^{2}}+\frac{\partial^{2} u_{i}}{\partial y^{2}}-k_{i}^{2} u_{i}=0, \quad(x, y) \in \Omega_{i}, \quad(i=1,2) \\
\frac{\partial u_{1}(-a, y)}{\partial x}-\beta u_{1}(-a, y)=0,\left.\quad u_{2}(x, y)\right|_{(x, y) \in C}=0 \\
u_{1}(0, y)=u_{2}(0, y), \quad \lambda_{1} \frac{\partial u_{1}(0, y)}{\partial x}=\lambda_{2} \frac{\partial u_{2}(0, y)}{\partial x}
\end{gathered}
$$




$$
u_{i}(x, 0)=0, \quad \frac{\partial u_{i}(x, \pi)}{\partial y}=0, \quad(i=1,2)
$$

is posed on $\Omega$.

The entries $\Gamma_{i j}(x, y ; \xi, \eta)$ in the matrix of Green's type

$$
\Gamma=\left(\Gamma_{i j}(x, y ; \xi, \eta)\right)_{i, j=\overline{1,2}}
$$

for the above problem setting are expressed, for a fixed location $\left(\xi^{*}, \eta^{*}\right)$ of the source point, as

$$
\Gamma_{i j}\left(x, y ; \xi^{*}, \eta^{*}\right)=G_{i j}\left(x, y ; \xi^{*}, \eta^{*}\right)+G_{i j}^{*}(x, y),
$$

where $G_{i j}\left(x, y ; \xi^{*}, \eta^{*}\right)$ represents the $i j$-th entry in the matrix of Green's type presented by Eq.(7). Clearly, in the entries $\Gamma_{11}$ and $\Gamma_{21}$, the source point $\left(\xi^{*}, \eta^{*}\right) \epsilon$ $\Omega_{1}$, whereas in the entries $\Gamma_{12}$ and $\Gamma_{22}$, it belongs to $\Omega_{2}$.

We introduce a circular fictitious contour $F=\left\{x^{2}+(y-\pi)^{2}=(\pi+\varepsilon)^{2}\right\}$ (with $0<x, y<\pi+\varepsilon)$ concentric with $C$, and express the first row entries $G_{1 j}^{*}(x, y),(j=$ $1,2)$ in the additive component $G^{*}(x, y)$ in Eq.(15) as the modified potential

$$
G_{1 j}^{*}(x, y)=\int_{F} G_{12}(x, y ; \xi, \eta) \mu_{j}(\xi, \eta) d F(\xi, \eta), \quad(x, y) \in \Omega_{1}
$$

while for the second row entries $G_{2 j}^{*}(x, y),(j=1,2)$ we have

$$
G_{2 j}^{*}(x, y)=\int_{F} G_{22}(x, y ; \xi, \eta) \mu_{j}(\xi, \eta) d F(\xi, \eta), \quad(x, y) \in \Omega_{2}
$$

The density functions $\mu_{j}(\xi, \eta),(j=1,2)$ in the above representation can be obtained by satisfying the boundary condition imposed on $C$. This yields the functional equations

$$
-G_{2 j}\left(x, y ; \xi^{*}, \eta^{*}\right)=\int_{F} G_{22}(x, y ; \xi, \eta) \mu_{j}(\xi, \eta) d F(\xi, \eta), \quad(x, y) \in C, \quad(j=1,2)
$$

in the density functions.

In Fig. 3 we depict profiles of the entries $\Gamma_{11}\left(x, y ; \xi^{*}, \eta^{*}\right)$ (defined for $(x, y) \in \Omega_{1}$ ) and $\Gamma_{21}\left(x, y ; \xi^{*}, \eta^{*}\right)$ (defined for $\left.(x, y) \in \Omega_{2}\right)$ in the matrix of Green's type that is found, where the source point is fixed at $(-.75,1.8) \in \Omega_{1}$. Parameters defining the statement were chosen as $k_{1}=0, k_{2}=1, \beta=0$, and $\lambda=10$. The series in Eq.(7) have been truncated to the $N$-th partial sum. Numerical solution of the functional equation in (16) and computation of values of $G_{1 j}^{*}(x, y)$ and $G_{2 j}^{*}(x, y)$ have been conducted by replacement of the line integrals in compliance with the standard 
trapezoid rule (with $m$ uniformly spaced quadrature nodes). Regularizing values of the computational parameters have been found as: $N=100, m=20$, and $\varepsilon=0.02$.

Fig.4 exhibits profiles of the entries $\Gamma_{12}\left(x, y ; \xi^{*}, \eta^{*}\right)$, with $(x, y) \in \Omega_{1}$, and $\Gamma_{22}\left(x, y ; \xi^{*}, \eta^{*}\right)$, with $(x, y) \in \Omega_{2}$, where the sounce point is fixed at $(1.0,1.8) \in \Omega_{2}$. Parameters defining the statement were chosen as $k_{1}=0, k_{2}=1, \beta=0$, and $\lambda=0.1$. Regularizing values of the computational parameters were found as: $N=100, m=20$, and $\varepsilon=0.01$.

Example 3.4: We consider a half-plane $\Omega=\{0<r<\infty, 0<\varphi<\pi\}$ composed of two fragments $\Omega_{1}=\{0<r<a, 0<\varphi<\pi\}$ and $\Omega_{2}=\{a<r<\infty, 0<\varphi<\pi\}$ filled in with different materials $\left(\lambda_{1}\right.$ and $\left.\lambda_{2}\right)$, with $r=a$ being the interface. The half-plane is weakened with a cut-off $\Omega_{3}$ whose contour $C$ represents a semi-ellipse, with semi-axes $\alpha_{0}$ and $\beta_{0}$, centered at $\left(a-\alpha_{0}, 0\right)$. Hence, $C$ internally touches the interface $r=a$ at $(a, 0)$ and its Cartesian equation appears as

$$
y=\frac{\beta_{0}}{\alpha_{0}} \sqrt{\alpha_{0}^{2}-\left[x-\left(a-\alpha_{0}\right)\right]^{2}}
$$

The matrix of Green's type

$$
\Gamma=\left(\Gamma_{i j}(r, \varphi ; \rho, \psi)\right)_{i, j=\overline{1,2}}
$$

is to compute now for the following boundary-contact value problem

$$
\begin{gathered}
\frac{1}{r} \frac{\partial}{\partial r}\left(r \frac{\partial u_{1}(r, \varphi)}{\partial r}\right)+\frac{1}{r^{2}} \frac{\partial^{2} u_{1}(r, \varphi)}{\partial \varphi^{2}}=0, \quad(r, \varphi) \in \Omega_{1} \backslash \Omega_{3} \\
\frac{1}{r} \frac{\partial}{\partial r}\left(r \frac{\partial u_{2}(r, \varphi)}{\partial r}\right)+\frac{1}{r^{2}} \frac{\partial^{2} u_{2}(r, \varphi)}{\partial \varphi^{2}}=0, \quad(r, \varphi) \in \Omega_{2} \\
u_{1}(a, \varphi)=u_{2}(a, \varphi), \frac{\partial u_{1}(a, \varphi)}{\partial r}=\lambda \frac{\partial u_{2}(a, \varphi)}{\partial r} \\
u_{1}(r, 0)=u_{2}(r, 0)=u_{1}(r, \pi)=u_{2}(r, \pi)=0,\left.\quad u_{1}\right|_{(r, \varphi) \in C}=0
\end{gathered}
$$

posed on the compound region $\widetilde{\Omega}=\Omega_{1} \backslash \Omega_{3} \cup \Omega_{2}$ of irregular shape.

We express the matrix $\Gamma$, for a fixed location $\left(\rho^{*}, \psi^{*}\right)$ of the source point, as

$$
\Gamma\left(r, \varphi ; \rho^{*}, \psi^{*}\right)=G\left(r, \varphi ; \rho^{*}, \psi^{*}\right)+G^{*}(r, \varphi),
$$

where $G\left(r, \varphi ; \rho^{*}, \psi^{*}\right)$ represents the matrix of Green's type for the compound halfplane, whose entries are presented by Eqs.(3)-(6).

To be certain, let $\left(\rho^{*}, \psi^{*}\right) \in \Omega_{2}$ and express the entries $G_{i 2}^{*}(r, \varphi),(i=1,2)$ of the first column of the additive component $G^{*}(r, \varphi)$ in Eq.(19) as

$$
G_{i 2}^{*}(r, \varphi)=\int_{F} G_{i 2}(r, \varphi ; \rho, \psi) \mu(\rho, \psi) d F(\rho, \psi), \quad(r, \varphi) \in \Omega_{i},
$$


where $F$ is a fictitious contour

$$
\left[x-\left(a-\alpha_{0}\right)\right]^{2}+y^{2}=\left(m_{0}-\varepsilon\right)^{2}
$$

concentric with $C$, with $m_{0}=\min \left(\alpha_{0}, \beta_{0}\right)$ and $\varepsilon$ representing a regularizing parameter.

The density function $\mu(\rho, \psi)$ in (20) can be obtained by satisfying the boundary condition imposed on $C$ for $u_{1}(r, \varphi)$. This yields the functional equation

$$
-G_{12}\left(r, \varphi ; \rho^{*}, \psi^{*}\right)=\int_{F} G_{11}(r, \varphi ; \rho, \psi) \mu(\rho, \psi) d F(\rho, \psi), \quad(r, \varphi) \in C
$$

in the density function $\mu(\rho, \psi)$.

In Fig.5 we depict profiles of the entries $\Gamma_{12}\left(r, \varphi ; \rho^{*}, \psi^{*}\right)$ and $\Gamma_{22}\left(r, \varphi ; \rho^{*}, \psi^{*}\right)$, which are defined on $\Omega_{1} \backslash \Omega_{3}$ and $\Omega_{2}$, respectively, in the matrix of Green's type for $\widetilde{\Omega}$, with $a=1$. The relative material conductivity is defined with $\lambda=0.2$, and the source point is fixed at $(1.25,2 \pi / 3) \in \Omega_{2}$. The cut-off's semi-axes are chosen as: $\alpha_{0}=0.5$ and $\beta_{0}=0.6$. Numerical routine based on the standard trapezoid rule was used in solving the functional equation in (21) and in computing values of the potential in Eq.(20). Regularizing values of the computational parameters have been found as: $m=20$, and $\varepsilon=0.02$.

\section{Multiply connected regions}

We will explore the potential of our approach to the construction of Green's functions by extending it to multiply connected regions of irregular configuration. Mixed boundary value problems will be of our interest as those with different types of boundary conditions imposed on different sections of the region's contour. Green's functions and matrices of Green's type for regions of standard shape (of the kind enlisted in Section 2) will be used to comprise kernels of resolving modified integal representations. Similarly to the presentation in Section 3, a set of particular examples will be presented below, each of which highlights different peculiarities of the approach and reveals an extent to which it is productive.

Example 4.1: We begin with computing the Green's function for the boundary value problem

$$
\begin{gathered}
\frac{\partial u}{\partial x}-\left.\beta u\right|_{x=0}=\left.u\right|_{y=x-1}=\left.u\right|_{y=0}=\left.\frac{\partial u}{\partial y}\right|_{y=1}=0, \quad \beta \geq 0 \\
\left.u\right|_{C}=0, \quad C=\left\{\left(x-x_{0}\right)^{2}+\left(y-y_{0}\right)^{2}=R^{2}\right\}
\end{gathered}
$$


posed for the Laplace equation in a double connected region $\Omega$ representing the trapezoid (shown in Fig.6) weakened with a circular aperture, with radius $R$, centered at $\left(x_{0}, y_{0}\right)$.

We express the Green's function $\Gamma\left(x, y ; \xi^{*}, \eta^{*}\right)$ of the boundary value problem in Eqs. (22) and (23) in the form

$$
\Gamma\left(x, y ; \xi^{*}, \eta^{*}\right)=G\left(x, y ; \xi^{*}, \eta^{*}\right)+G^{*}(x, y),
$$

where the source point $\left(\xi^{*}, \eta^{*}\right)$ is arbitrarily fixed inside $\Omega$. The component $G\left(x, y ; \xi^{*}, \eta^{*}\right)$ represents in (24) the Green's function for the solid semi-strip (see Eq.(1) in Section 2) and brings the logarithmic singularity to $\Gamma\left(x, y ; \xi^{*}, \eta^{*}\right)$ at $(x, y)=\left(\xi^{*}, \eta^{*}\right)$, if the component $G^{*}(x, y)$ is harmonic at any point $(x, y)$ in $\Omega$.

For $\Gamma\left(x, y ; \xi^{*}, \eta^{*}\right)$ to comply with the relations in Eqs. (23) and (22), $G^{*}(x, y)$ ought to satisfy the conditions imposed on $x=0, y=0$, and $y=1$, while on $y=x-1$ and on $C$ it ought to satisfy the conditions

$$
G^{*}(x, x-1)=-G\left(x, x-1 ; \xi^{*}, \eta^{*}\right)
$$

and

$$
G^{*}(x, \widetilde{y}(x))=-G\left(x, \widetilde{y}(x) ; \xi^{*}, \eta^{*}\right)
$$

where $\widetilde{y}(x)=y_{0} \pm \sqrt{R^{2}-\left(x-x_{0}\right)^{2}}$

Since $G^{*}(x, y)$ is harmonic in $\Omega$, to find it we construct the modified integral representation

$$
G^{*}(x, y)=\sum_{i=1}^{2} \int_{F_{i}} G(x, y ; \xi, \eta) \mu_{i}(\xi, \eta) d F_{i}(\xi, \eta),
$$

where the fictitious contour $F_{1}$ is a segment of the straight line $y=x-\left(1+\varepsilon_{1}\right)$ ranging from 0 to 1 , while $F_{2}$ is a circle, with radius $R-\varepsilon_{2}$, concentric with $C$. Notice that $\varepsilon_{1}$ and $\varepsilon_{2}$ represent regularizing parameters.

Upon satisfying the conditions in (25) and (26), we obtain the following system of functional equations

$$
\begin{aligned}
& -G\left(x, x-1 ; \xi^{*}, \eta^{*}\right)=\sum_{i=1}^{2} \int_{F_{i}} G(x, x-1 ; \xi, \eta) \mu_{i}(\xi, \eta) d F_{i}(\xi, \eta) \\
& -G\left(x, \widetilde{y}(x) ; \xi^{*}, \eta^{*}\right)=\sum_{i=1}^{2} \int_{F_{i}} G(x, \widetilde{y}(x) ; \xi, \eta) \mu_{i}(\xi, \eta) d F_{i}(\xi, \eta)
\end{aligned}
$$

in the density functions $\mu_{1}(\xi, \eta)$ and $\mu_{2}(\xi, \eta)$. After the latter functions are found, the representation in $(27)$ is used to compute the profile $\Gamma\left(x, y ; \xi^{*}, \eta^{*}\right)$ of the Green's function in $\Omega$. 
Fig.6 shows a contourplot of $\Gamma\left(x, y ; \xi^{*}, \eta^{*}\right)$ where the parameters in the setting are: $\beta=2.0, x_{0}=0.4, y_{0}=0.3$, and $R=0.15$, while the source point $\left(\xi^{*}, \eta^{*}\right)$ was fixed at $(0.8,0.6)$. The regularizing values of the parameters $\varepsilon_{1}$ and $\varepsilon_{2}$ were, in this case, found as 0.03 and 0.05 , respectively. The standard trapezoidal rule $(m=20)$ was used to numerically solve the system of functional equations in $\mu_{1}(\xi, \eta)$ and $\mu_{2}(\xi, \eta)$ as well as to compute values of the integral representation in Eq.(27).

With the next example, which is more or less trivial, our intent is to explore the potential of our algorithm toward problem settings with different kinds of boundary conditions imposed on contours of apertures in multiply connected regions.

Example 4.2: In doing so, consider a double connected region $\Omega$ representing a half-plane $\{y>0\}$ weakened with a hole whose contour $C$ is a circle, with radius $a$, centered at $\left(x_{0}, y_{0}\right)$. We will compute a profile $\Gamma\left(x, y ; \xi^{*}, \eta^{*}\right)$ of the Green's function for the mixed boundary value problem

$$
\begin{gathered}
\frac{\partial^{2} u(x, y)}{\partial x^{2}}+\frac{\partial^{2} u(x, y)}{\partial x^{2}}=0, \quad(x, y) \in \Omega \\
u(x, 0)=0,\left.\quad \frac{\partial u(x, y)}{\partial n}\right|_{(x, y) \in C}=0,
\end{gathered}
$$

where a source point $\left(\xi^{*}, \eta^{*}\right)$ is arbitrarily located in $\Omega$ and

$$
n=\cos (n, x) \frac{\partial}{\partial x}+\cos (n, y) \frac{\partial}{\partial y}
$$

represents the normal direction to $C$.

As usual, we look for the Green's function in the form

$$
\Gamma\left(x, y ; \xi^{*}, \eta^{*}\right)=G\left(x, y ; \xi^{*}, \eta^{*}\right)+G^{*}(x, y)
$$

where

$$
G\left(x, y ; \xi^{*}, \eta^{*}\right)=\frac{1}{2 \pi} \ln \sqrt{\frac{\left(x-\xi^{*}\right)^{2}+\left(y+\eta^{*}\right)^{2}}{\left(x-\xi^{*}\right)^{2}+\left(y-\eta^{*}\right)^{2}}}
$$

represents the classical [1] Green's function of the Dirichlet problem for the halfplane. The component $G^{*}(x, y)$ is expressed with a modified integral representation as

$$
G^{*}(x, y)=\int_{F} G(x, y ; \xi, \eta) \mu(\xi, \eta) d F(\xi, \eta)
$$

where the fictitious contour is a circle

$$
F=\left\{\left(x-x_{0}\right)^{2}+\left(y-y_{0}\right)^{2}=(a-\varepsilon)^{2}\right\}
$$

concentric to $C$, with $\varepsilon$ representing a regularization parameter. 
Since the sets of observation and source points never overlap, the Neumann condition on $C$ results in the following regular functional equation

$$
-\frac{\partial G\left(x, y ; \xi^{*}, \eta^{*}\right)}{\partial n}=\int_{F} \frac{\partial G(x, y ; \xi, \eta)}{\partial n} \mu(\xi, \eta) d F(\xi, \eta), \quad(x, y) \in C
$$

in the density function $\mu(\xi, \eta)$. No computational obstacles arise while solving this equation numerically.

To illustrate the effectiveness of our approach, the profile $\Gamma(x, y ; 0,3.5)$ of the Green's function that we have computed is depicted in Fig.7, with $x_{0}=2.0$, $y_{0}=2.0, \beta=2.0$, and $a=1.0$. The regularizing value for $\varepsilon$ was found, in this case, as 0.2 .

The example that follows is designed to show that the number of apertures in multiply connected regions, their shape, location, and type of boundary conditions imposed on them do not represent a factor in our algorithm.

Example 4.3: A triple connected region $\Omega$ represents the semi-strip $\{0<x<$ $\infty, 0<y<b\}$ weakened with an elliptic aperture contoured with

$$
C_{1}=\left\{\frac{\left(x-x_{1}\right)^{2}}{\alpha_{1}^{2}}+\frac{\left(y-y_{1}\right)^{2}}{\beta_{1}^{2}}=1\right\}
$$

and the circular aperture

$$
C_{2}=\left\{\left(x-x_{2}\right)^{2}+\left(y-y_{2}\right)^{2}=a_{2}^{2}\right\}
$$

Mixed boundary value problem for Laplace equation is considered where Dirichlet and Neumann conditions are imposed on $C_{1}$ and $C_{2}$, respectively, while the boundary conditions on the outer contour of $\Omega$ are defined by Eq.(22).

The Green's function $\Gamma\left(x, y ; \xi^{*}, \eta^{*}\right)$ for the above setting is expressed by the integral representation in Eq.(24) where the fictitious contours $F_{1}$ and $F_{2}$ are, in this case, defined as

$$
F_{1}=\left\{\left(x-x_{1}\right)^{2}+\left(y-y_{1}\right)^{2}=\left(m_{1}-\varepsilon_{1}\right)^{2}\right.
$$

and

$$
F_{2}=\left\{\left(x-x_{2}\right)^{2}+\left(y-y_{2}\right)^{2}=\left(a_{2}-\varepsilon_{2}\right)^{2},\right.
$$

where $m_{1}=\min \left(\alpha_{1}, \beta_{1}\right)$, while $\varepsilon_{1}$ and $\varepsilon_{2}$ represent regularizing parameters.

By satisfying the boundary conditions on $C_{1}$ and $C_{2}$, we obtain the following system of resolving functional equations

$$
-G\left(x, y ; \xi^{*}, \eta^{*}\right)=\sum_{i=1}^{2} \int_{F_{i}} G(x, y ; \xi, \eta) \mu_{i}(\xi, \eta) d F_{i}(\xi, \eta), \quad(x, y) \in C_{1}
$$




$$
-\frac{\partial G\left(x, y ; \xi^{*}, \eta^{*}\right)}{\partial n}=\sum_{i=1}^{2} \int_{F_{i}} \frac{\partial G(x, y ; \xi, \eta)}{\partial n} \mu_{i}(\xi, \eta) d F_{i}(\xi, \eta), \quad(x, y) \in C_{2}
$$

The profile $\Gamma(x, y ; 0.8,0.5)$ of the Green's function, as depicted in Fig.8, has been obtained by using our customary algorithm. The parameters defining the setting has been defined as: $b=1.0, x_{1}=0.35, y_{1}=0.5, \alpha_{1}=0.12, \beta_{1}=0.1, x_{2}=1.3$, $y_{2}=0.5$, and $a_{2}=0.2$. Regularizing values of the computational parameters were found as: $N=100, m=20, \varepsilon_{1}=0.02$, and $\varepsilon_{2}=0.04$.

The following two examples are presented to just show that our numerical algorithm ensures high accuracy level in computing Green's functions in compound multiply connected regions when contours of apertures either touch or even cross interfaces of materials.

Example 4.4: The half-plane $\{0<r<\infty, 0<\varphi<\pi\}$ is considered as composed of two fragments $\Omega_{1}=\{0<r<a, 0<\varphi<\pi\}$ and $\Omega_{2}=\{a<r<\infty, 0<$ $\varphi<\pi\}$ filled in with different materials $(\lambda=0.1)$. The fragment $\Omega_{2}$ is weakened with a circular aperture whose contour $C$ touches the interface line $r=a$. This generates a double connected compound region $\Omega$. Dirichlet boundary condition is assumed on the inner contour $C$ of $\Omega$.

The matrix, with entries presented in Eqs.(3)-(6), has been employed for obtaining the kernel of the resolving inregral representation, which we used to compute the regular components of the matrix $\Gamma(x, y ; \xi, \eta)$ of Green's type for the double connected region $\Omega$. Fig. 9 presents the profiles $\Gamma_{11}(r, \varphi ; 0.5, \pi / 3)$ and $\Gamma_{21}(r, \varphi ; 0.5, \pi / 3)$ of the first column entries in this matrix.

Example 4.5: The infinite strip $\{-\infty<x<\infty, 0<y<b\}$ is composed of three fragments $\Omega_{1}=\{-\infty<x<-a, 0<y<b\}, \Omega_{2}=\{-a<x<a, 0<y<b\}$, and $\Omega_{3}=\{a<x<\infty, 0<y<b\}$, each of which is filled in with materials of different conductive properties $\left(\lambda_{i},(i=1,2,3)\right)$. The strip and weakened by the "fat circle" shaped aperture

$$
C=\left\{\left(x-x_{0}\right)^{4}+\left(y-y_{0}\right)^{4}=a_{0}^{4}\right\}
$$

whose center is on the interface line of $\Omega_{2}$ and $\Omega_{3}$, forming a double connected compound region $\Omega$.

Our algorithm has been used for computing the matrix $\Gamma(x, y ; \xi, \eta)$ of Green's type for $\Omega$. The matrix defined in Eq.(8) has been employed in obtaining the kernel in the resolving potential representation for the regular components in $\Gamma(x, y ; \xi, \eta)$. Three entries of this matrix are depicted in Fig.10. Since the source point $\left(\xi^{*}, \eta^{*}\right)$ is located in $\Omega_{1}$, these entries are $\Gamma_{i 1}\left(x, y ; \xi^{*}, \eta^{*}\right),(i=1,2,3)$. The relative material conductivities are defined with $\Lambda_{1}=\Lambda_{2}=0.1$. 


\section{Closure}

We have explored, in this study, the potential of the Green's function modification of the method of functional equations in computing Green's functions and matrices of Green's type for mixed boundary value problems stated on 2-D regions of irregular configuration. Laplace and Klein-Gordon equation have been considered. Computer-friendly forms of Green's functions for regions of standard shape have been presented and employed in our computer algorithm. Thus, this study widens the range of computer implementations for the Green's function methods in applied sciences.

\section{Acknowledgment}

We acknowledge the Summer 2005 Faculty Research Grant at Middle Tennessee State University, which radically enhanced our work on this project.

\section{References}

[1] R. Courant and D. Hilbert, Methods of Mathematical Physics, vol. 2, Intersciences, New York, 1962.

[2] V.D. Kupradze, Potential Method in the Theory of Elasticity, Davey, New York, 1965.

[3] Y.A. Melnikov, Influence functions of a point source in regions of complex configuration, Computational Mechanics, vol.23, 5, pp.304-315, 1996.

[4] Y.A. Melnikov, Influence Functions and Matrices, Marcel Dekker, New York-Basel, 1998.

[5] D. G. Duffy, Green's Functions with Applications, CRC Press, Boca Raton, 2001.

[6] M.A. Sales and L.J. Gray, Evaluation of the anisotropic Green's function and its derivative, Comp. Struct., 69, pp.247-254, 1998.

[7] W.T. Ang and J.C.F. Telles, A numerical Green's function for multiple cracks in anisotropic bodies, J. Eng. Maths, vol.49, 3, pp.197-207, 2004.

[8] E. Pan and F. Han, Green's functions for transversely isotropic piezoelectric multilayered half-spaces, J. Eng. Maths, vol.49, 3, pp.271-278, 2004.

[9] V.K. Tewary, Elastostatic Green's function for advanced materials subject to surface loading, J. Eng. Maths, vol.49, 3, pp.289-304, 2004. 
Y.A. Melnikov and M.Y. Melnikov / Electronic Journal of Boundary Elements, Vol. 4, No. 3, pp. 82-104 (2006)

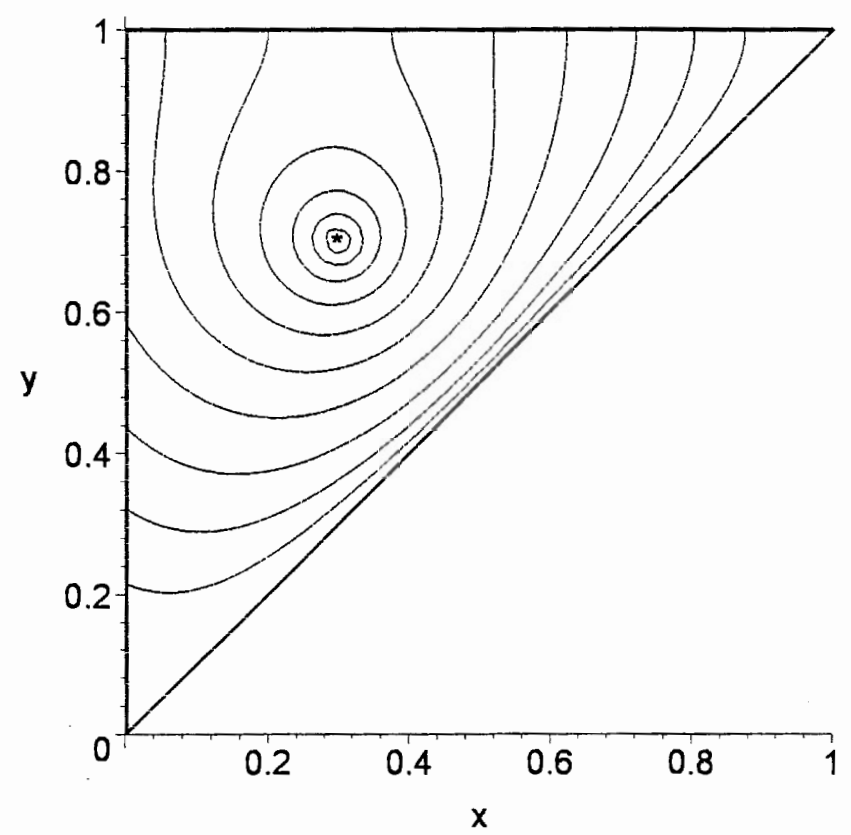

Figure 1: Green's function of a mixed problem in a triangular region

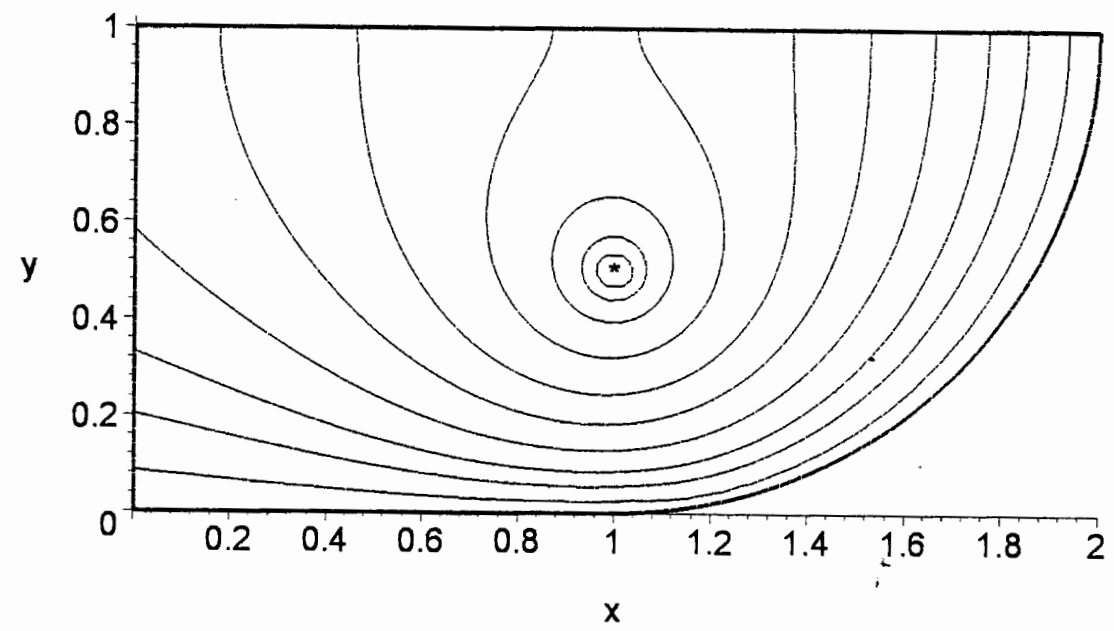

Figure 2: Profile of the Green's function in a region of irregular shape 


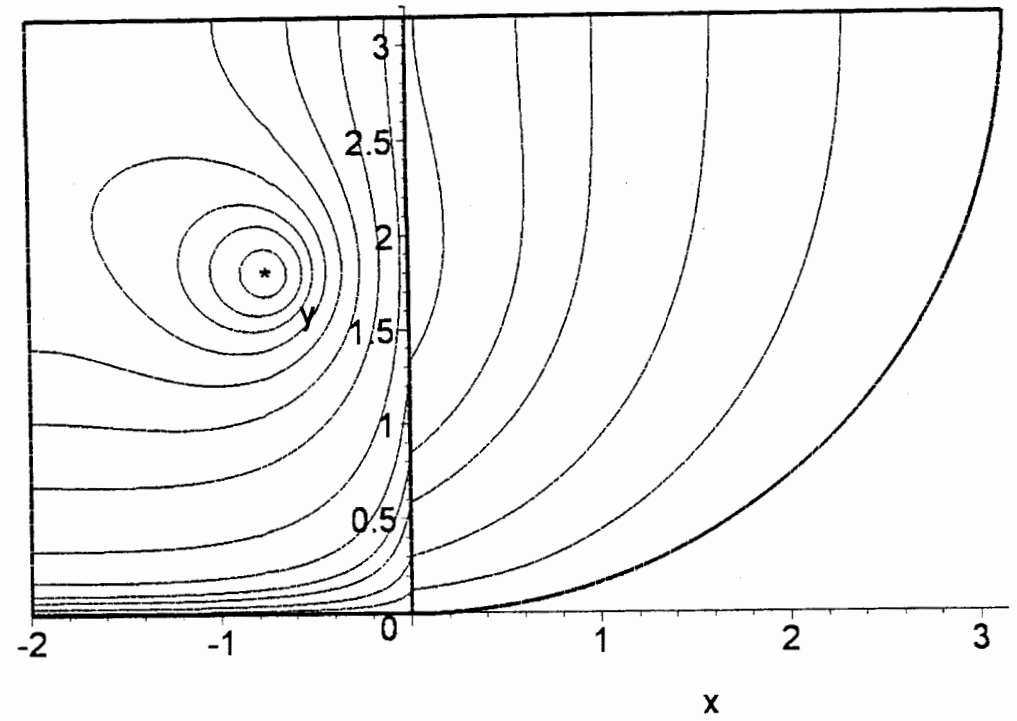

Figure 3: Entries of the first column in the matrix of Green's type

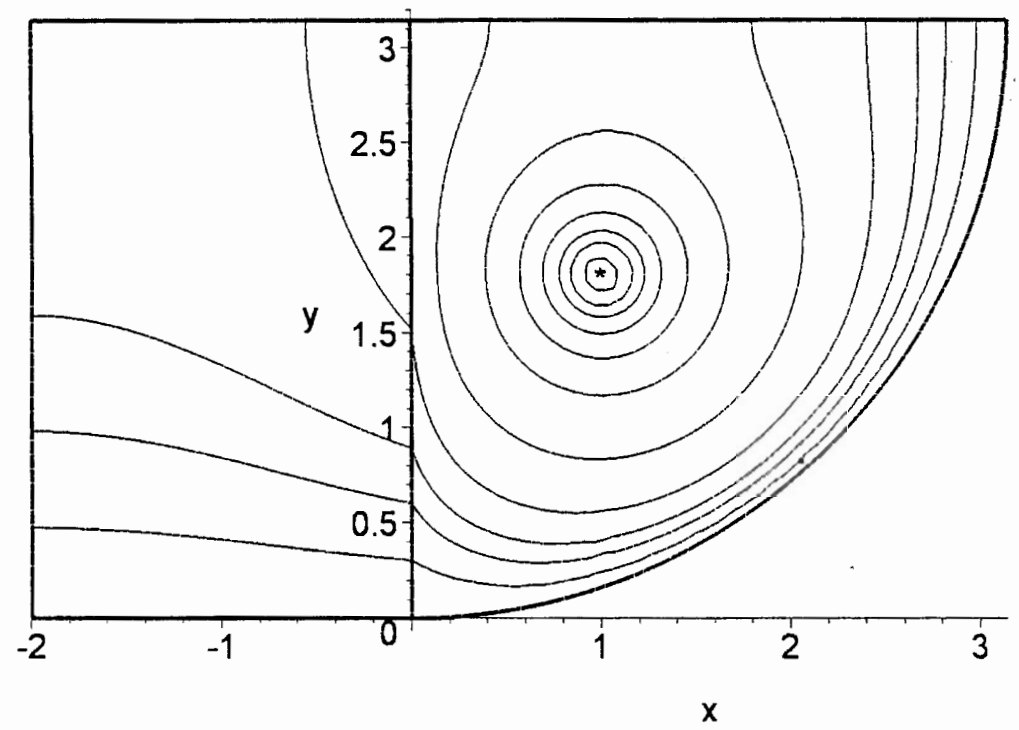

Figure 4: Entries of the second column in the matrix of Green's type 


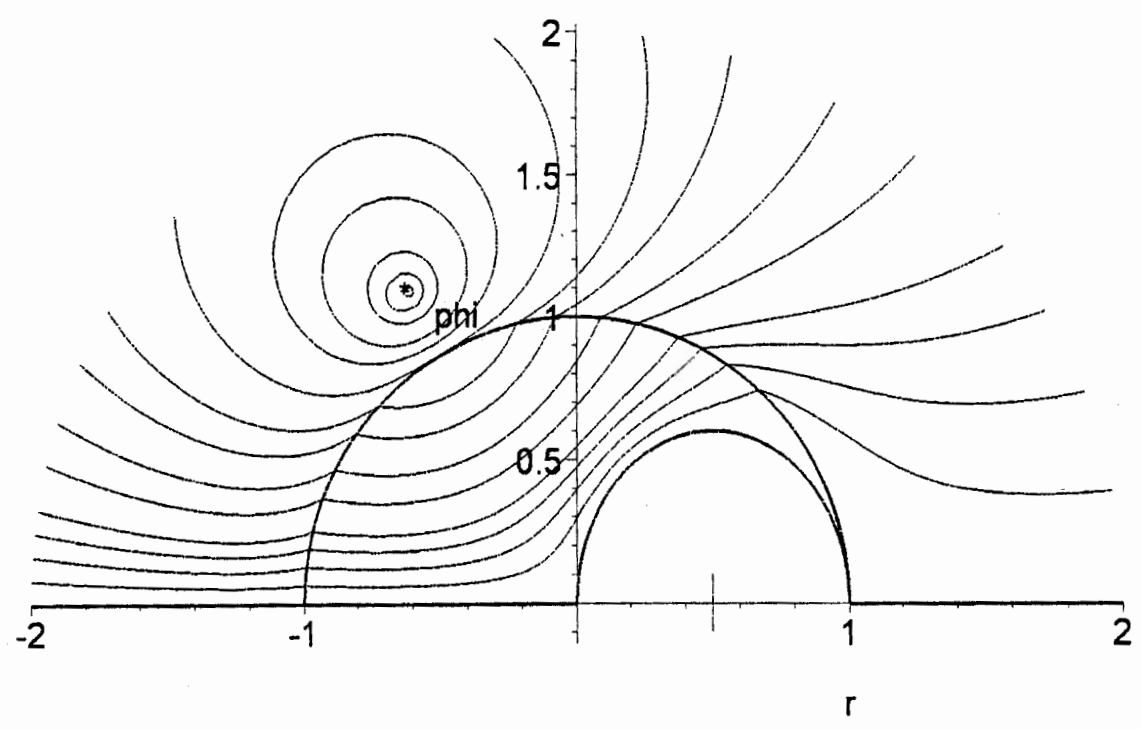

Figure 5: Compound half-plane weakened with an elliptic cut-off

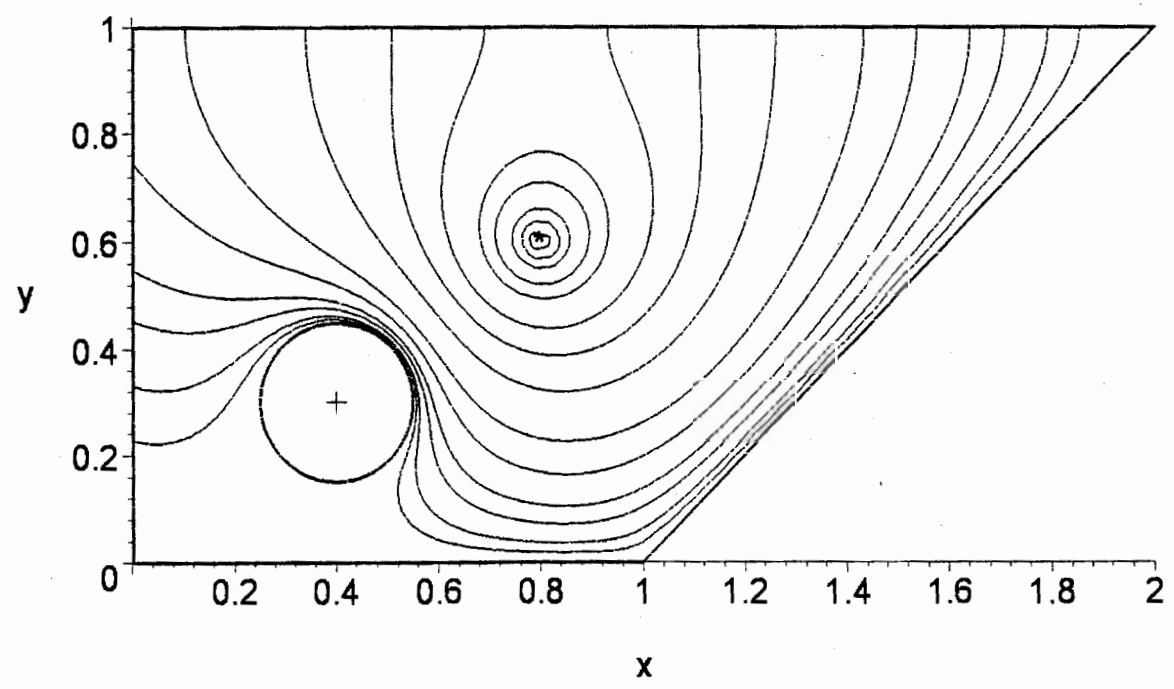

Figure 6: Profile of the Green's function in a double connected region 


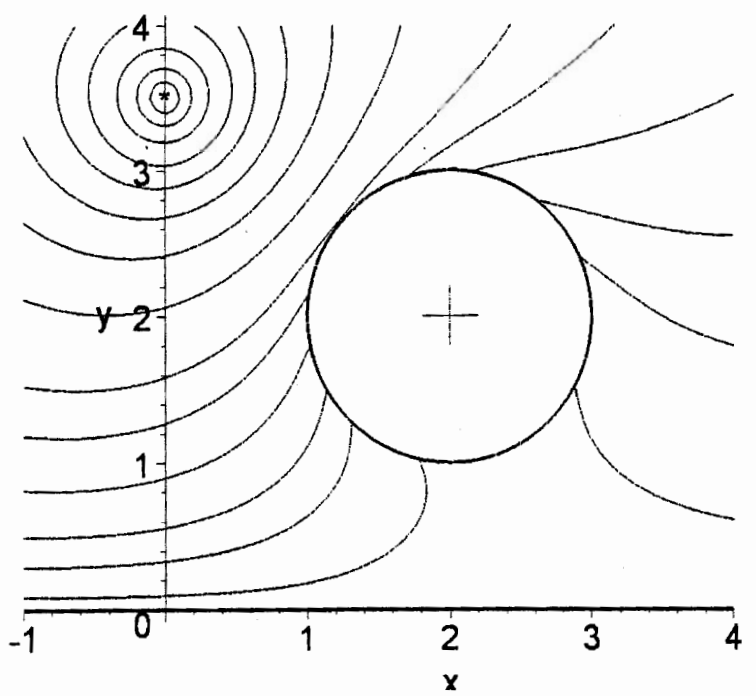

Figure 7: Robin boundary conditions imposed on the aperture

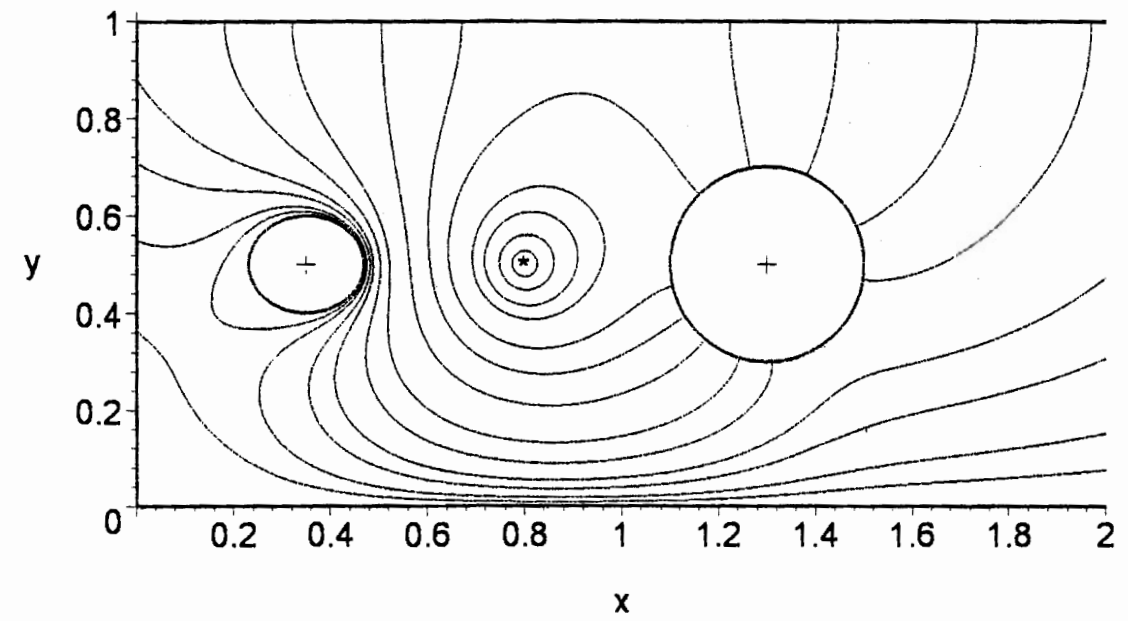

Figure 8: Mixed problem in a multiply connected region 


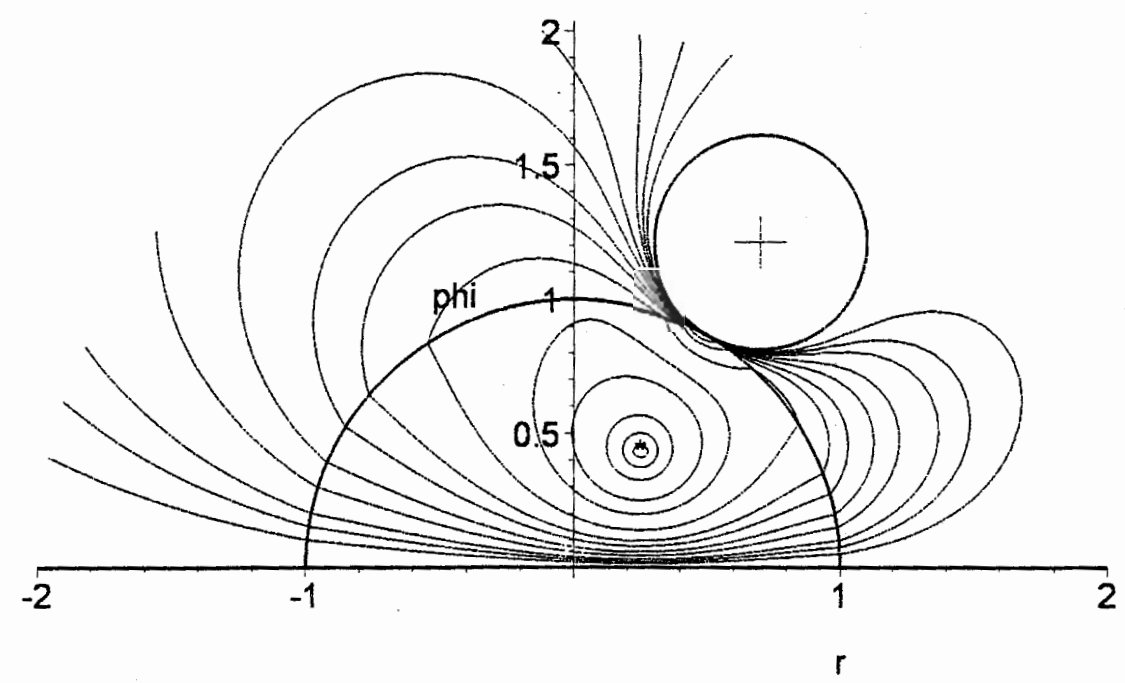

Figure 9: Aperture touching the interface line

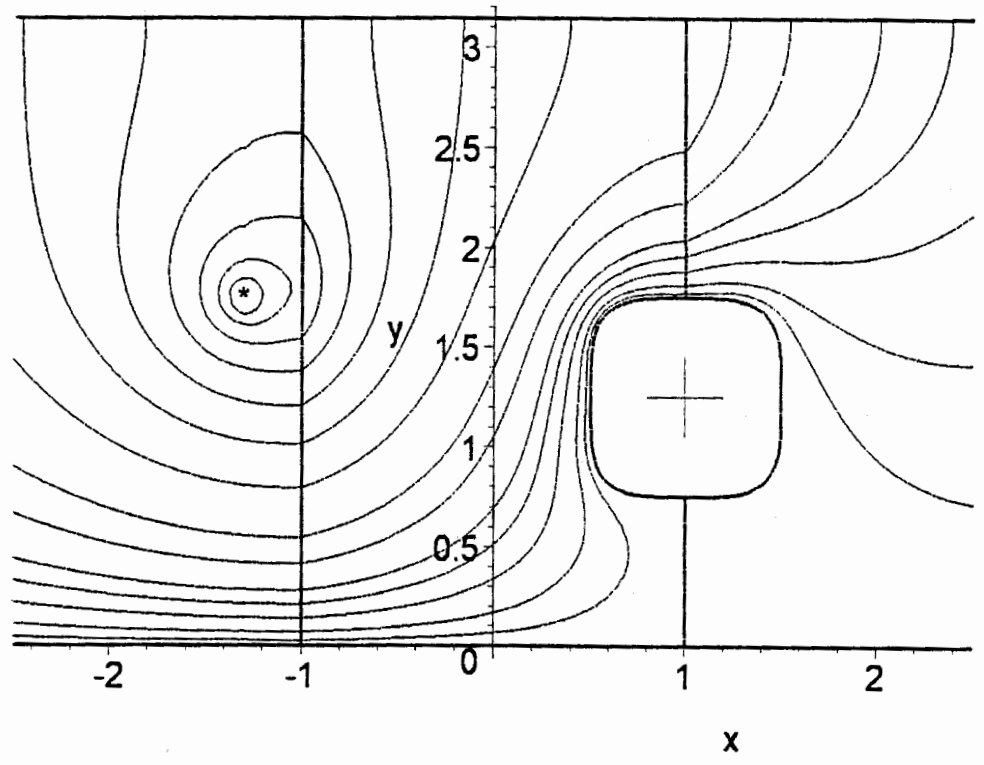

Figure 10: Contour of an aperture crosses the interface line 